\title{
The Importance of Continuing Solar System-Wide Impact Crater Studies
}

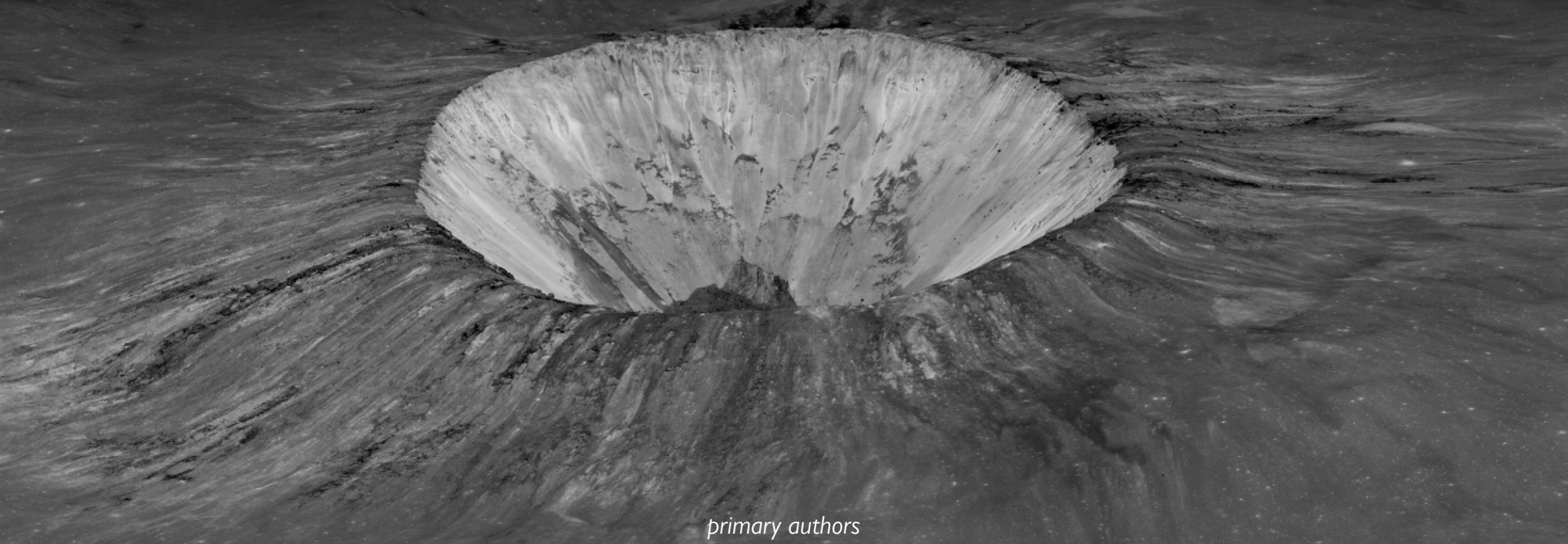

Stuart J. Robbins Southwest Research Institute - stuart@boulder.swri.edu - (303)-918-5589

Michelle R. Kirchoff Southwest Research Institute - kirchoff@boulder.swriedu

\author{
co-authors \\ Nadine G. Barlow (Northern Arizona University), Clark R. Chapman (Southwest Research Institute), \\ Simone Marchi (Southwest Research Institute), Elizabeth A. Silber (Western University), \\ and Angela M. Stickle (The Johns Hopkins University Applied Physics Laboratory)
}

endorsers / co-signers

Jennifer L. B. Anderson, Chimira N. Andres, Maria E. Banks, Olivier Barnouin, Daniel C. Berman, Edward Bierhaus, Joseph M. Boyce, Donald M. Burt, Veronica J. Bray, Mark J. Cintala, Christopher J. Cline II, Bob Craddock, David A. Crawford, Ingrid Daubar, Luke Dones, Brian Enke, Ronald A. Fevig, Travis S.J. Gabriel, James W. Head, Ken Herkenhoff, Rachael R. Hoover, Ya Huei Huang, Jose M. Hurtado Jr., Abderrahmane Ibhi, Thomas Kenkmann, David T. King Jr., Edwin Kite, Christian Klimczak, Christian Koeberl, Graham Lau, Anna Losiak, Alice Lucchetti, Elena Martellato, Matteo Massironi, Moses P. Milazzo, Yasunori Miura, Miki Nakajima, Michael C. Nolan, Jessica Noviello, Jens Ormö, Lillian R. Ostrach, Maurizio Pajola, Samantha Peel, Divya M. Persaud, Cynthia Phillips, Jennifer Piatek, Vidhya G. Rangarajan, Jamie D. Riggs, Frances Rivera-Hernández, Christoph M. Schäfer, Paul Schenk, Katherine Shirley, Kelsi N. Singer, David E. Stillman, Marshall J. Styczinski, Seiji Sugita, Jessica M. Sunshine, Anne Verbiscer, Wes A. Watters, Jean-Pierre Williams, Paul F. Wren, Michael F. Zeilnhofer, and Nicolle Zellner 
Key Findings

- Impact crater studies are a fundamental part of nearly all solid body exploration across the Solar System.

- Continued investigations of impact craters are a key component for all future planetary missions.

- Continued funding of impact crater studies, including field work, laboratory and computer simulations, and for related fields that could assist crater studies such as artificial intelligence is critical.

\section{Introduction, and the Important Science Questions}

Impact cratering is ubiquitous across the Solar System, and impact craters are seen on practically all well resolved solid bodies. Impact craters form via an almost instantaneous release of energy when a small body (the impactor) strikes a larger body (the target). These events and the signs they leave are critical to our understanding of the Solar System in many ways, and the purpose of this white paper is to support their continued study over the next decade.

Impact craters (hereafter "craters") are important to understand population statistics, for craters directly relate to the impactor population. When craters form, the excavated cavity depends on the energy of the impactor. Assuming a probability distribution for impact angle and speed, the primary remaining variable is kinetic energy, directly relatable to the impactor's mass through experimental and numerical scaling laws. Therefore, the size distribution of craters can be related back to the impactor population. Because craters are $10-20 \times$ larger than the impactors that created them, observing kilometer-scale craters can inform studies about smaller solar system bodies that have eluded telescopic detection. It is also important to understand the evolution of impactor populations over time, and hence the dynamical evolution of the Solar System.

Craters are important for understanding solar system chronology, and several papers to this Decadal Survey emphasize this need (Cohen et al., Ghent et al., Schenk et al.). Without radiometric samples for absolute dating, craters are the only method we have to quantitatively understand ages of features on a planetary body: If a surface is older than another, it will have more craters because it has had a longer time to accumulate them; with stratigraphy, crater spatial densities yield relative ages. Tying crater spatial densities to absolute dates via radiometric dating of rocks from that surface enables development of an absolute chronology model. All crater-based chronology models have uncertainties, so better understanding and calibration of crater-based chronologies should be an important part of the next decade's research. This especially includes efforts to monitor for new craters forming via impact flashes (Moon) and repeat, meter-level imaging (Moon, Mars).

Craters, as individual structures, are fundamental for studying planetary geology. When a crater forms, excavating a cavity below the surrounding surface, nature has done our drilling: Craters expose layers in the subsurface, giving a view of the crustal history of bodies throughout the Solar System and the composition of materials that would be otherwise buried. Also, to a first order, craters form with predictable shapes that vary with size. However, other geologic factors, such as the presence of target volatiles, surface erosion, isostasy, and subsurface layering that may be present during or following crater formation can affect the shape of these craters. A more detailed understanding of original crater shape, and the shapes produced by different geologic factors and what caused them, should provide a significant improvement in the ability to use crater morphology to determine geologic processes that produced modified crater shapes, and their history.

More research is needed in the next decade to understand the processes that form craters, through observations, laboratory experiments, and numerical simulations. Fundamental questions remain about crater formation, such as how central peaks and scalloped rims form, how very large basins form, how material is excavated, and how secondary craters result.

Finally, craters form on all solid bodies in the Solar System, and so any study of planetary surfaces must consider the effects of cratering. The study of crater populations and features would be greatly assisted through faster ways to identify them via artificial intelligence, which has progressed 
but still is inadequate for most cratering-related investigations. We have organized this paper by how data are gathered so that they can be better placed into the context of future missions.

\section{The Study of Impact Craters by Remote Sensing}

Remote sensing spacecraft are typically the cheapest and easiest missions to fly, not requiring any landing, sampling, nor return of material to Earth. Fortunately, remote sensing observations provide several kinds of key data for understanding craters.

\subsection{Importance of Flyby and Orbiter Data}

Luna 3's first images in 1959 of the lunar far side revealed a completely different landscape from the near side, and later missions greatly increased our understanding of the Moon and its dominant landform: craters. Flyby missions to every planet in the Solar System, numerous asteroids, comets, and Kuiper Belt objects (KBOs), have all increased our understanding of craters and of planetary stratigraphy and chronology. However, information is still incomplete because of the highly variable pixel scale, incomplete coverage, and other limitations or absence data types such as topography. Despite this, we include flyby science because flybys remain the most common mission flown. The New Horizons flyby mission, for example, revolutionized our understanding of small KBOs based on the crater populations KBOs produced on Charon and Pluto (Singer et al., 2019).

Orbiters offer much improved imaging campaigns compared with flybys. They permit repeat coverage by numerous instruments and cross-validation of datasets. For example, stereo-based topography (using the principle of parallax from two or more images) might be the only method to return elevation data during a flyby. However, with an orbiter, a laser altimeter can be used to tie that topography to an absolute reference frame and provide long-baseline corrections, such as identifying giant basins. This was done with no fewer than data from five missions to Mercury, the Moon, Mars, Eros, and Bennu, and the laser topography data have significantly improved our understanding of crater formation, modification, and scaling laws between bodies with different gravities and surface materials. Also, improved image coverage from orbiters allows one to gather more crater statistics from each body and to identify and understand those craters that might not represent the general population. Unique crater forms are important for understanding unique environments on each body. So far, only the Inner Solar System planets, a few asteroids, Saturnian satellites, a comet, and to a lesser extent Jovian satellites have been studied by orbiters.

Basic imaging - usually in clear filters for better signal-to-noise - is important for understanding the basics and most common applications of cratering: impactor populations and ages. High resolution color imaging/spectroscopy is important to understand what material craters bring up from depth and how craters have altered their surroundings. Finally, topography is necessary to understand how craters have physically altered their surroundings and how geological processes have altered the craters. The best coverage is currently for Mars and the Moon, while good coverage exists for Mercury, Ceres, Vesta, Eros, Bennu, comet 67P, Itokawa, and Ryugu. We have less data of less variety for Venus and bodies farther from the Sun - especially for the Uranian and Neptunian satellites. Data and conclusions based on the Inner Solar System and Asteroid Belt cannot be easily extrapolated to the rest of the Solar System due to completely different dynamical environments. To truly understand cratering as a process throughout the Solar System and the implications for small and large body evolution, we need these data for all relevant bodies from Mercury, out to the satellites of the giant planets, and beyond into the Kuiper Belt.

\subsection{Imaging Strategies for Limited Data}

Many missions cannot provide an ideal instrument suite and data-taking campaigns due to practical issues, often related to cost, mass, and energy limitations. Given this reality, we provide recommendations for optimizing the science return for crater studies with limited instrumentation. 
If foreknowledge exists, missions will often target interesting and unique terrain. While such terrain will often have craters, to understand how craters evolve on a body, how that terrain might have modified them, and understand the population of impactors that struck the body, additional targeting of relatively "clean" surfaces devoid of other features is important. Terrain that does not have more complex geologic features is ideal for studying crater populations and impact-related geology, for the craters can be observed without other complicating factors. For example, the lunar maria and Mercury's smooth plains are more ideal for studying craters on those bodies, for there are relatively few complicating features. With these "clean" surfaces, the population of craters can be better understood, craters' topography can be better constrained, and mineralogical signatures can be tied to the crater and the layers it reveals. Therefore, we recommend that future missions include targeting of these terrains-if possible-to further crater studies.

A strategy to maximize science from flyby imaging is to have nested images of the same terrain at varying pixel scales. This strategy can be executed as the spacecraft's distance to the target varies: When far away, global images are taken, and as the craft gets closer, successively better pixel scale images can be obtained. For example, New Horizons executed this at Pluto and Charon, providing hemispheric coverage at up to $\sim 1 \mathrm{~km} / \mathrm{pix}$, but nested coverage at up to $\sim 160 \mathrm{~m} / \mathrm{pix}$ at Charon and $\sim 80 \mathrm{~m} /$ pix at Pluto. This sort of coverage is important for understanding how craters form at various scales, the context of smaller features within the broader landscape, and the population of craters from which the population of impactors can be derived. Before New Horizons, our only direct knowledge of the population of $\mathrm{KBOs}$ was through Earth telescopic observations of KBOs larger than $\sim 15 \mathrm{~km}$, and stellar occultation detections of KBOs $100 \mathrm{~m}$ across, a gap of two orders of magnitude. Pluto's and Charon's crater record covered this critical range, and they revealed unexpected dynamic structure in the Kuiper Belt (Singer et al., 2019). Such a significant science return would not have been possible with just hemispheric low-resolution imaging or localized high-resolution imaging, and required the nested imaging on Charon's smooth plains.

Finally, we emphasize that imaging campaigns optimized for stereo topography reconstruction can greatly improve the science return of any mission, especially flybys. No flyby mission has ever included a laser altimeter, so the only way to reconstruct topography is through photoclinometry ("shape-from-shading") and stereo. The former technique is useful for relatively small features, but it becomes unstable without any anchor at larger scales. That anchor can be provided by stereo, which operates through the principle of parallax. Topography allows testing of cratering scaling laws, understanding how deep material might have been brought up from the subsurface, volume displacement, and other studies critical to understanding craters as features in and of themselves.

\subsection{Additional Remote Sensing Data}

After initial survey data are complete through flyby or lower-cost orbiter missions, secondary instruments that could not be selected for initial survey missions can be used for critical follow-up data that allow further understanding of craters as an integrated system with the surrounding planetary body. These types of instruments include high-resolution (spatially and spectrally) spectrometers, cameras at other wavelengths beyond visual (e.g., far IR, UV, microwave), radar and radio reflectors/sounders, and laser altimeters.

For example, the CRISM instrument, a high-resolution spectral and spatial spectrometer on Mars Reconnaissance Orbiter, revolutionized our understanding of Martian surface mineralogy, including material only observed in craters, despite plenty of previous-generation spectrometers flown. Similarly, the inclusion of mini-RF as a technology demonstration on the Lunar Reconnaissance Orbiter (LRO) allowed us to understand the lunar surface in radio wavelengths at high spatial scales, which enhanced our understanding of crater ejecta distribution. Also, Diviner on LRO allowed for detailed understanding of thermal inertia, which has led to a new understanding of recent lunar 
chronology and cratering (Ghent et al., 2014; Mazrouei et al., 2019). The MESSENGER mission to Mercury that followed up from Mariner 10 included radar reflectivity and a laser altimeter, which enhanced the study of the shapes of craters. Including these sorts of instruments on future missions would substantially improve our understanding of craters and how they affect planetary surfaces.

\section{The Study of Impact Craters through in situ Methods}

In situ methods can provide data that are impossible to gather from flybys and orbiters, such as detailed exploration of geology and chemistry (e.g., Mars rovers) and precise formation and modification ages of craters (e.g., Apollo). There are four types of common in situ methods: touchand-go, stationary landers, mobile vehicles, and human. The two most often thought of to provide the most and best information are mobile vehicles and human exploration; in this paper, we will only address landers and mobile vehicles because touch-and-go data are minimally useful for craters, and human exploration is covered in a different Decadal Survey.

To-date, the only reliable absolute ages we have of craters are from the extensive study of the Apollo and Luna mission samples. In addition, the best information we have of detailed crater geology not observed by Apollo astronauts is from the Mars rovers Spirit, Opportunity, and Curiosity. Researchers are working on ways to use stationary and mobile vehicles to get absolute ages either in situ or as robotic sample returns (Cohen et al., this decadal survey). This is necessary because the capability to send humans safely throughout the Solar System will not exist for a long time.

\subsection{Stationary Landers}

As remote analysis of surface chemistry and mineralogy continues to advance, the ability of stationary landers to provide informative data for cratering on many bodies improves. Landers have the advantage of going to bodies where mobility is limited, especially given technology constraints within the next decade (e.g., rough terrains, Venus, comets, or icy heavily cratered bodies), and mass and power constraints for larger instruments (e.g., in situ radiometric dating). These landers provide very detailed and thorough studies of a single area. Given data limitations on bodies other than Earth, Moon, and Mars, even the analysis of one crater on another planetary surface, especially in the Outer Solar System, would provide information vital to understanding cratering in different environments (e.g., Venus) and materials (e.g., extremely cold ice). Such investigations would enhance the study of craters as geologic features and what they can tell us about the subsurface as revealed by ejecta and crater walls.

Advances in in situ radiometric dating (e.g., Cohen et al., this decadal survey) of key, stratigraphically distinct impact sites would significantly advance our understanding of the impact flux, regardless of the planetary body on which the measurement is made. Even the lunar cratervs-age relationship is still poorly understood (e.g., Ghent et al., this decadal survey), while no other body has even a single datum that can tie crater spatial densities to an absolute age reference. Without absolute ages, the timing and duration of geologic processes is relegated to the accuracy of dynamical models and all the assumptions built into those models.

\subsection{Mobile Vehicles (Hoppers, Rovers, and Aircraft)}

The science return of a mobile-instead of stationary-laboratory can significantly enhance the science return of a mission, including crater science. The extraordinary success of the Opportunity rover, which visited numerous craters on its $45.16 \mathrm{~km}$ journey, demonstrated that even in that relatively small area of Mars, many different stratigraphic layers were visible in different crater walls, exposing different eras in Martian history, and different mineralogy. Such a mobile science platform deployed elsewhere-either by a rover system or various in-development hopper systems-could similarly increase the return of any future mission.

Now-novel technologies that NASA has only recently selected for flight-the dual-blade Ingenuity 
for Mars and dual quadcopter Dragonfly for Titan-have the potential to increase our understanding of craters even more, at least on bodies with an atmosphere to provide flight. Flight can significantly improve ground coverage, and it can allow the best of both worlds of an orbiter and rover: touching down to allow in situ measurements and lifting off and providing an aerial view of the larger region. In addition, observations from such technologies provide a platform for observations intermediate to those from orbit and from the ground. For example, magnetic and gravity surveys, whose resolution depends on distance from the surface, could provide important insights into the subsurface structure of the craters and crater formation on other planetary bodies. Due to the significant potential for increasing the breadth and variety of terrain explored, these vehicles are important for improving our understanding of craters and development should be continued.

\section{The Study of Impact Craters through Laboratory Experiments and Simulations, and Detection Advancements}

\subsection{Laboratory Experiments}

Laboratory cratering experiments have been used since the Apollo era to provide insights into cratering physics. Experiments are vital for understanding cratering physics because they show what happens following an impact and do not require a priori physical models. A few specialized facilities exist around the world-including two at NASA centers-to perform impact experiments at speeds, and with materials, relevant to planetary cratering. These facilities have provided data vital in understanding impact crater formation and comparisons to morphologies seen on a variety of planetary surfaces. Other, smaller, facilities have been developed at other institutions, but often their capabilities are more limited than NASA facilities, or they are designed to study other aspects of cratering like material failure. It is vital to support these new facilities and that support for the NASA impact facilities continue in the next decade to better understand the impact process.

Laboratory-scale experiments have energies several orders of magnitude smaller than natural, macroscopic cratering events. Despite the wide difference in scale, laboratory experiments provide insights into fundamental physics that occur during and following impact, formation of crater shapes, how target and projectile properties affect the cratering process and resultant crater, and data about kinetic impactors-vital for planetary defense. Experiments provide the required information to develop impact process scaling laws, which are analytic expressions for a variety of crater properties (e.g., Holsapple, 1993). These analytic expressions relate observables (principally crater size and depth) to the parameters of the collisions, such as impactor size, density, and velocity. These scaling relationships are used widely in the community. However, the applicability of these rules is limited, including for oblique impacts and impacts into many solar system materials (e.g., ices). Further experiments are necessary to develop more robust scaling relationships for the variety of impacts we see across the Solar System. Dedicated campaigns for the development of material physics and equation of state models are also critical, for these powerful tools are necessary for the modeling and interpretation of experimental, numerical, and observational data.

Laboratory tests and the relatively recent advent of high-speed photography have similarly allowed the detailed study of how impact craters form in different materials-including solid metal for the upcoming Psyche mission, or loose ice-rock for the 2005 Deep Impact mission. High-speed images are used to study fractures and fragmentation, impact flash, and physics of the ejecta curtain. Increases in imaging capability in the past decade have revolutionized our ability to track the highspeed response of materials, and continued support is necessary to ensure high-speed diagnostics can continue to be used in experiments. Measurements and observations extend beyond images, and we suggest that investment opportunities for a variety of high-speed diagnostics be made in the next decade to improve our understanding of cratering processes at impact facilities. 
Impact experiments represent our only easily accessible, real-life example of what really happens during and after an impact. Combining insights from experiments with field observations and numerical simulations is the only way to get the full view of impact cratering processes.

\subsection{Numerical Simulations}

The scale of large craters across the Solar System is prohibitive to experimentally reproduce; other factors such as gravity and some specific materials are also challenging to reproduce experimentally, which could be especially important in later stages of crater formation.

Numerical models provide an important method to study formation of large-scale impact structures and associated processes, and modeling works hand-in-hand with, and complements, observations and laboratory experiments to provide a glimpse into dynamics of impact processes at all scales. Significant development of shock-physics codes used for cratering studies over the last few decades has increased their usability as tools for crater research (e.g., McGlaun et al., 1990; Owen et al., 1998; Collins et al., 2004), but much remains to be done. Many of the most commonly used codes include a variety of sophisticated descriptions of material behavior, including high temperature and pressure behavior (parameterized by the Equation of State) and strength response (represented by the plasticity model) (e.g., Pierazzo et al., 2008).

It is critical that more accurate, robust, and publicly-available material models for relevant geologic materials continue to be developed and adopted into numerical codes, which would improve the accuracy of the simulations. Experimental campaigns and code development go handin-hand, for are both needed to accurately represent the high temperature and pressure behavior of the wide variety solar system materials (e.g., ice types, meteorite compositions, common rock classes), which differ drastically from engineering materials (e.g., aluminum) that have benefitted from extensive previous and ongoing study. Effectively, if we cannot accurately simulate craters we see on planetary bodies, then we do not yet fully understand how they form and are modified. Continued opportunities to propose impact modeling studies to R\&A programs is vital in order to continue to allow this valuable toolset to increase our understanding of craters.

Additionally, simulations of impact cratering can rapidly become computationally prohibitive. Continued optimization and inclusion of new solvers and techniques could greatly enhance the usability of codes and thus greatly increase our understanding of impact cratering processes.

\subsection{Artificial Intelligence (AI) Detection of Craters}

Another consideration is from fields of study that might seem unrelated to impact craters, but that can be used to enhance the field, as initially noted above with better equation solvers. A prime additional example is from the burgeoning field of $\mathrm{Al}$, which uses machine learning techniques to identify features-much like a human learning that a square block cannot go into a round hole.

The primary application of machine learning's ability to identify craters is for the science of impact crater populations. Crater populations are important to understand, as described earlier in this white paper, but doing so requires identifying and measuring the craters. There are hundreds of millions-if not larger orders of magnitude-impact craters visible in existing spacecraft imagery, let alone what will be collected over the coming decade. While manually constructed crater databases exist for hundreds up to millions of craters, the amount of imagery and manual time required for identification quickly becomes prohibitive. Al detection of craters to build these databases, or even assist in manual construction, would be a significant boon for crater population studies, and it deserves continued research funds.

\subsection{Terrestrial Field Work}

Finally, an important aspect of craters is they do form on Earth, and we can study them through terrestrial field work. Field work allows significantly more detailed studies of craters, such as ejecta 
deposition, uplift studies, and fracturing of the crust. Field campaigns to terrestrial craters provide important benchmarking and insights to better understand numerical and laboratory models (e.g., Morgan et al., 2016). However, there are still significant questions to be answered that field work could inform, so we support its funding through the Planetary Sciences Division of NASA.

\section{Summary}

Cratering is one of the most predominant processes in the Solar System. Solar System bodies display size distributions dominated by smaller bodies: planetesimals, asteroids, comets, and circumplanetary debris. When such impactors encounter larger bodies, they explode, creating impact craters. Except for very geologically active bodies like lo and Earth, the older surfaces of nearly all solar system bodies are heavily cratered. Craters range from microscopic pits to giant, multi-ring basins thousands of kilometers across.

Spatial densities of craters on surface units permit absolute geologic time to be extrapolated (for the few bodies from which we have meteorites and returned samples), via modeling of the dynamical evolution of small bodies, to planets, satellites, asteroids, KBOs, etc. throughout the Solar System, establishing the interplanetary correlation of geologic time. Where absolute ages are difficult to measure or estimate, differences in crater spatial densities provide at least relative stratigraphic ages. The morphologies of craters show how various geologic processes, including cratering itself, degrades topography with time.

Impact craters also provide probes, analogous to road-cuts on Earth, into the surfaces and crusts of Solar System bodies, permitting studies of regolith and megaregolith development, volcanic and lacustrine depositional processes, and erosional and tectonic processes. To thoroughly understand what craters reveal about the geological history of planets and smaller bodies, we must study in detail how craters form and evolve, using in situ studies of individual impact craters on different bodies (including terrestrial analogs), laboratory impact experiments, and hydrocode modeling of impact physics.

During the next decade, missions can be planned that use advanced remote sensing and in situ instruments synergistically with other investigations to broaden our knowledge of cratering on worlds not previously visited or at resolutions not previously attained. Continued development of computational and laboratory techniques should advance our ability to apply such new observations of craters to understanding of planetary processes and evolution. We strongly advocate for impact crater studies, and all they encompass, to continue to be funded in the coming years.

\section{References}

Cohen, B. et al. (2020) Geochronology for the next decade. white paper submitted to this Decadal Survey. II Collins, G.S. et al. (2004) Modeling damage and deformation in impact simulations. Meteoritics \& Planetary Science, $39(2)$, p. 217-231. doi:10.1111/j.1945-5100.2004.tb00337.x || Ghent, R.R. et al. (2014) Constraints on the recent rate of lunar ejecta breakdown and implications for crater ages. Geology, 42(12), p. 1059-1062. doi:10.1130/G35926.1 || Ghent, R.R. et al. (2020) Assessing the recent impact flux in the Inner Solar System: 1 Ga to present. white paper submitted to this Decadal Survey. II Holsapple, K. A. (1993). The scaling of impact processes in planetary sciences. Annual Review of Earth and Planetary Sciences, 21(1), p. 333-373. doi:10.1146/annurev.ea.21.050193.002001 || Mazrouei, S. et al. (2019) Earth and Moon impact flux increased at the end of the Paleozoic. Science, 363(6424), p. 253-257. doi:10.1126/science.aar4058 || McGlaun, J.M. et al. (1990). CTH: A three-dimensional shock wave physics code. International Journal of Impact Engineering, 10(1-4), p. 351-360. doi:10.1016/0734-743X(90)90071-3 || Morgan, J.V. et al. (2016) The formation of peak rings in large impact craters. Science, 354(6314), p. 878-882. doi:10.1126/science.aah6561 || Owen, J.M. et al. (1998) Adaptive smoothed particle hydrodynamics: Methodology. II. The Astrophysical Journal Supplement Series, 116(2), p. 155-209. doi:10.1086/313100 |l Pierazzo, E. et al. (2008) Validation of numerical codes for impact and explosion cratering: Impacts on strengthless and metal targets. Meteoritics \& Planetary Science, 43(12), p. 1917-1938. doi:10.1111/j.1945-5100.2008.tb00653.x || Schenk, P.M. et al. (2020) The chronology problem in the Outer Solar System: Constraining the "WHEN" of major dynamical and geological events. white paper submitted to this Decadal Survey. II Singer, K.N. et al. (2019) Impact craters on Pluto and Charon indicate a deficit of small Kuiper Belt objects. Science, 363(6430), p. 955-959. doi:10.1126/science.aap8628 